The International Journal of Indian Psychology

ISSN 2348-5396 (e) | ISSN: 2349-3429 (p)

Volume 5, Issue 1, DIP: 18.01.098/20170501

DOI: $10.25215 / 0501.098$

http://www.ijip.in | October-December, 2017

Original Research Paper

\title{
Standards of Lithium Treatment Monitoring In a Teaching Hospital in South India among Bipolar Affective Disorder
}

\author{
Dr. Sureshkumar Ramasamy ${ }^{1 *}$, Dr. Shilpa Srinivasan ${ }^{2}$, \\ Dr. Shree Aarthi Ramanathan ${ }^{3}$
}

\section{ABSTRACT}

Background: Lithium is a commonly used drug with a narrow therapeutic index, it has significant adverse effects on kidney and thyroid, and is life threatening during intoxication. Maudsley guidelines on managing bipolar affective disorder patients (BPAD) with lithium recommends checking weight at baseline and every 3rd month, serum lithium after 7th day thereafter 3 months once, renal function test and serum thyroid stimulating hormone (TSH) at baseline and 6 months once. Aim: To evaluate whether Pre-Lithium workup and monitoring is done in Bipolar affective disorder (BPAD) patients initiated on Lithium as per Maudsley prescribing guidelines in psychiatry. Method: Retrospective audit conducted in a tertiary care hospital on lithium monitoring in BPAD patients treated with lithium atleast for six months compared against Maudsley prescribing guidelines. Results: Among medical records of 114 bipolar patients fulfilling study criteria, weight and serum lithium at baseline was checked in $100 \%$ of patients; serum creatinine and TSH at baseline along with lithium at 3rd month was done in $83.3 \%, 78.9 \%$ and $68.4 \%$ of patients respectively. Maudsley guidelines for serum creatinine, TSH and lithium level at 6th month were met only in $43.8 \%, 43.8 \%$ and $72.8 \%$ of patients respectively. Conclusion: The quality of lithium monitoring in bipolar patients falls well short of accepted standards; Hence addressing the issues in monitoring and following a standard protocol can improve the effectiveness of treatment and quality of life of patients.

Keywords: Lithium Monitoring, Lithium, Audit, Bipolar Affective Disorder.

Bipolar affective disorder (BPAD) is a mood disorder characterized by episodes of mania, hypomania and depression and the lifetime prevalence ranges between $0.3 \%$ and $4.8 \%{ }^{[1]}$ Bipolar affective disorder is almost always recurrent. Lithium is approved by FDA and commonly used for treating mood disorders. Lithium is a drug with wide therapeutic applications but with a narrow therapeutic index. The accepted serum lithium level for

\footnotetext{
${ }^{1}$ Assistant Professor, Dept Of Psychiatry, PSG Medical College, Avinashi road, Coimbatore, Tamilnadu, India

${ }^{2}$ Senior Resident, Department Of Psychiatry, Shri Sathya Sai Medical College And Research Institute,

Kancheepuram, Tamilnadu, India.

${ }^{3}$ Consultant Psychiatrist, GR Hospital, Coimbatore, Tamilnadu, India

*Responding Author

(C) 2017 Ramasamy S, Srinivasan S, \& Ramanathan S A; licensee IJIP. This is an Open Access Research distributed under the terms of the Creative Commons Attribution License (www.creativecommons.org/licenses/by/2.0), which permits unrestricted use, distribution, and reproduction in any Medium, provided the original work is properly cited.
} 


\section{Standards of Lithium Treatment Monitoring In a Teaching Hospital in South India among Bipolar Affective Disorder}

treating patients is $0.6-1.2 \mathrm{meq} / \mathrm{L} .{ }^{[2]}$ Some of the common side effects encountered with lithium are weight gain, sedation, nausea, vomiting, diarrhea, tremors, acne, hair loss, dysphoria, reduced creativity, dysarthria, hypothyroidism, renal disease and during lithium toxicity patients may develop coarse tremor, dysarthria, ataxia, seizures, coma and death. Toxic effects of lithium become more likely as the serum level rises above $1.5 \mathrm{meq} / \mathrm{L} .{ }^{[3]} \mathrm{But}$ patients can develop lithium toxicity signs at any serum lithium levels depending on their tolerability. ${ }^{[2]}$ Up to $75 \%$ of patients treated with lithium experience some side effects. ${ }^{[4]}$ Hypothyroidism occurs in 5\% - 35\% of patients treated with lithium. It occurs more frequently in women. ${ }^{[5,6]}$ Approximately $10 \%-20 \%$ of patients receiving long term lithium treatment display morphological kidney changes, usually interstitial fibrosis, tubular atrophy and sometimes glomerular sclerosis. ${ }^{[7]}$

Appropriate baseline assessment prior to lithium treatment will identify patients at significant risk. Also periodic monitoring will help us for early detection of side effects thereby preventing any serious complications. Maudsley prescribing guidelines is one among the various standard guidelines which is accepted widely for monitoring patients on lithium.

The National Institute for Health and Care Excellence (NICE) defines clinical audit as a 'quality improvement process that seeks to improve patient care and outcomes through systematic review of care against explicit criteria and the implementation of change'. By auditing clinical practice against standards derived from evidence-based guidelines, defined aspects of the quality of care delivered to patients can be measured and monitored. ${ }^{[8]}$ For trainees, participation in audit can allow a number of intended learning outcomes to be achieved, these include gaining an understanding of how clinical governance can be applied in practice, and the development of leadership skills related to monitoring performance and effecting change. ${ }^{[9]}$

Hence we wanted to evaluate whether a standard protocol is being followed in our set up for monitoring patients on lithium treatment.

Aim:

- To evaluate whether Pre-Lithium workup and monitoring is done in Bipolar affective disorder (BPAD) patients initiated on Lithium as per Maudsley prescribing guidelines in psychiatry.

\section{MATERIALS \& METHOD}

\section{Study Design \& Participants}

This was a retrospective study looking at pre lithium workup and lithium monitoring in bipolar affective disorder patients treated with lithium. This study was conducted in a tertiary care medical college hospital in India. We screened medical records of all patients registered in psychiatry department in our hospital between January 2011 and March 2014. We included both out-patients and in-patients aged between 18 years and 65 years, who were 


\section{Standards of Lithium Treatment Monitoring In a Teaching Hospital in South India among Bipolar Affective Disorder}

diagnosed to have bipolar affective disorder as per international classification of disease $10^{\text {th }}$ edition (ICD-10). We included only those patients who were started on tablet lithium by the department of psychiatry in our hospital and followed up in our department for at least 6 months duration after initiating lithium.

We excluded patients whose diagnosis is other than bipolar affective disorder and who are not on lithium. We also excluded patients who were already on lithium before visiting our department or patients who were started on lithium by an outside psychiatrist. Among the 451 medical records retrieved with a diagnosis of bipolar affective disorder, 114 patients fulfilled the inclusion and exclusion criteria and were recruited for further analysis. Information were gathered regarding age, gender, primary diagnosis, baseline values of weight, serum creatinine, serum thyroid stimulating hormone level (TSH) and periodic monitoring of serum Lithium, serum creatinine and TSH up to 6 months using a data collection form. In case of any discrepancy regarding the information recorded in the file, it was sorted out after discussion between the authors.

\section{Materials:}

Maudsley prescribing guidelines in psychiatry ${ }^{[10]}$ is a standard evidence based and widely accepted guidelines, which provides clinicians with practical and useful advice on prescribing psychotropic agents in commonly encountered clinical situation.

Maudsley guidelines on monitoring patients on lithium recommends checking weight at baseline, $3^{\text {rd }}$ and $6^{\text {th }}$ month, serum lithium to be checked after $7^{\text {th }}$ day of starting lithium or change of dose thereafter every 3 months, estimated glomerular filtration rate (eGFR) and serum thyroid stimulating hormone (TSH) to be done at baseline and 6 months once.

The gathered information was compared with the Maudsley guidelines for identifying whether the baseline work up and periodic monitoring of laboratory values that were carried out during that period was optimal or not. For the purpose of this study we have slightly modified Maudsely guidelines as in instead of eGFR we have chosen serum creatinine levels, as eGFR is not done in our set up.

The study was approved by Institutional human ethics committee.

\section{Statistical analysis:}

Each of the four outcomes of interest (measures of serum lithium level, serum creatinine, thyroid stimulating hormone and body weight) was measured dichotomously; whether or not the standard had been met. Percentage of people who underwent these assessments was calculated. The data was entered and analyzed using Microsoft Excel worksheet for windows 7. 


\section{Standards of Lithium Treatment Monitoring In a Teaching Hospital in South India among Bipolar Affective Disorder}

\section{RESULTS}

A total of 114 medical records fulfilling study criteria were identified and reviewed to check whether standard protocol is followed while monitoring patients on lithium.

The mean age of people in our study was 35.3 years (age range: 18 - 62 years) and sample predominantly consisted of male patients $(\mathrm{N}=41 ; 65 \%)$.

Base line weight was checked in $97.3 \%$ of patients, weight at $3^{\text {rd }}$ and $6^{\text {th }}$ month as well as serum lithium at baseline was done in all patients, serum lithium at $3^{\text {rd }}$ month $\& 6^{\text {th }}$ month was done only in $68.4 \%$ \& $72.8 \%$ of patients respectively, serum creatinine at baseline \& at $6^{\text {th }}$ month was checked in $83.3 \%$ \& $43.8 \%$ of patients respectively and serum TSH levels at baseline $\&$ at $6^{\text {th }}$ month was done only in $78.9 \%$ \& $43.8 \%$ of patients respectively (Table 1).

Table 1: Percentage of people in whom monitoring was carried out as per Maudsley guidelines

\begin{tabular}{|l|c|c|c|c|c|c|}
\hline \multirow{2}{*}{$\mathbf{N}=114$} & \multicolumn{2}{|l|}{ Baseline } & \multicolumn{2}{l|}{$\mathbf{3}^{\text {rd }}$ month } & \multicolumn{2}{l|}{$\mathbf{6}^{\text {th }}$ month } \\
\cline { 2 - 7 } & $\mathbf{n} / \mathbf{N}$ & $\mathbf{0}$ & $\mathbf{n} / \mathbf{N}$ & $\%$ & $\mathbf{n} / \mathbf{N}$ & $\%$ \\
\hline Weight & $111 / 114$ & $97.3 \%$ & $114 / 114$ & $100 \%$ & $114 / 114$ & $100 \%$ \\
\hline Serum Lithium & $114 / 114$ & $100 \%$ & $78 / 114$ & $68.4 \%$ & $83 / 114$ & $72.8 \%$ \\
\hline Serum Creatinine & $95 / 114$ & $83.3 \%$ & & & $50 / 114$ & $43.8 \%$ \\
\hline $\begin{array}{l}\text { Thyroid } \\
\text { stimulating } \\
\text { hormone (TSH) }\end{array}$ & $90 / 114$ & $78.9 \%$ & & & $50 / 114$ & $43.8 \%$ \\
\hline
\end{tabular}

\section{DISCUSSION}

Good doctors - Safer patients calls for the reinvigoration of clinical audit to support better patient care and service improvement. The Royal College of Psychiatrists' guidance emphasizes that it is not the direct involvement in data collection that is important, but rather the review of the audit evidence for the quality of care provided, and taking the lead in implementing strategies to improve quality where these are required. ${ }^{[9]}$ Such reflection on the data by clinicians is perhaps the most potent aspect of clinical audit. It is important to note that although audit can generate new knowledge, its primary purpose is to compare practice against pre-determined standards. To our knowledge, this is the first study done in India to audit lithium monitoring practice in BPAD patients receiving lithium. In this study, we selected only those patients who are diagnosed to have bipolar affective disorder, firstly as in these patients lithium is usually started not only for acute management as in $1^{\text {st }}$ episode of mania, but also as a prophylaxis to prevent future episodes wherein lithium is given for long duration. Secondly, recruiting bipolar affective disorder patients alone makes the sample homogenous, where a standard protocol can be followed. For the same reason, patients in extremes of age category were excluded, as these patients may require more frequent assessments and additional investigations. 


\section{Standards of Lithium Treatment Monitoring In a Teaching Hospital in South India among Bipolar Affective Disorder}

Though there are various standard guidelines for monitoring patients on lithium, Maudsley practice guideline appears much more simpler, but at the same time evidence based and feasible in our set up, given the fact that health care system especially mental health care in developing countries like India is below compared to developed countries like USA and UK. In our study, we found that weight is checked in $97.3 \%$ of patients at baseline and $100 \%$ during review which could be explained by the fact that it is easy to check and has been routinely done for all the patients irrespective of the diagnosis, and there is no cost involved. Serum lithium is done for all patients at baseline which is a welcome thing, but subsequently lithium level was not done for more than $1 / 4^{\text {th }}$ of the patients, the reason could be serum lithium at baseline is usually done to adjust the dosage rather than for monitoring side effects. Serum creatinine and TSH at baseline was done in around $80 \%$ of patients, but subsequent monitoring of these parameters at 6 month was carried out only in $43.8 \%$ of patients. Our study shows quality of lithium monitoring in patients falls well short of accepted standards and targets which could be compared with the similar findings noted in audits done in the west. An audit done in UK on lithium monitoring against recommended standard NICE guidelines in patients recently starting lithium, showed a documented baseline measure of renal or thyroid function in $84 \%$ and $82 \%$ respectively. For patients prescribed lithium for a year or more, the NICE standards for monitoring lithium serum levels, and renal and thyroid function were met in $30 \%, 55 \%$ and $50 \%$ of cases respectively. ${ }^{[11]}$ Despite the existence of explicit standards for monitoring patients who are prescribed lithium, a number of local audits conducted in different areas of the UK over the last 20 years have found this monitoring to be suboptimal. ${ }^{[12,13,14,15]}$ These findings suggest that there is no standard protocol which is being followed in monitoring patients on lithium treatment.

\section{Possible explanation for sub optimal monitoring:}

There could be various reasons for this like patient related factors and clinician related factors. Patient related factors:

Previous studies done in western countries have identified certain factors that may influence monitoring rates like willingness of patients to have blood tests and either receiving inadequate information about lithium treatment and the need for regular blood tests or not assimilating the information given, ${ }^{[16,17]}$ Other factors are patient not being compliant to treatment, unaffordability and patient unable to review on time due to personal reasons or simply forgetting to do the test.

\section{Clinician related factors:}

Certain clinician related factors that can influence lithium monitoring are clinician unable to spend adequate time with the patient, forgetting to ask for investigation, clinician's perception that patient may not do it; clinicians inadequate knowledge regarding standard protocols for monitoring patients on lithium or despite being aware of standard guidelines clinician may not feel any need to follow the guidelines. 


\section{Standards of Lithium Treatment Monitoring In a Teaching Hospital in South India among Bipolar Affective Disorder}

Ours being a tertiary care teaching hospital, where these standard guidelines are often discussed and taught to postgraduate students, the possibility that doctors not being aware of existence of any standard protocol is less likely. But looking at our findings which show a sub optimal standard of monitoring patients on lithium; it appears that theoretical knowledge has not actually translated into clinical practice, which could also be due to various logistic reasons.

Few clinicians may disagree with the recommendations given by Maudsley guidelines, as every patient is different and it is the clinical judgment based on one's own clinical experience that could help in treating these patients, rather than doing serum creatinine and TSH every 6 months. But we should keep in mind, in a country like India where people tend to ignore early symptoms leading to delayed identification of even common medical illness like diabetes; there is always a possibility that one can miss early warning signs of serious adverse effects. Also, given the fact that lithium can cause serious side effects and has a narrow therapeutic index following a standard protocol is worthwhile.

Our findings cannot be generalized to other set up, however one may not be absolutely wrong in assuming that the findings might be similar in other tertiary or non-tertiary care centers in India.

Strengths of the study are the topic itself which needs to be addressed, homogenous sample wherein a standard protocol can be followed, using a simple and feasible guidelines and auditing helps to improve the clinical service provided.

Limitations of the study are the retrospective design of the study itself, relying solely on medical records to collect details (in our study, missing reports and nil documentation were taken as "test not done"), small sample size which is due to strict selection criteria in our study, short term study - however, looking at the findings one can easily assume that the rate of monitoring must be similar or much worse after 1 to 2 years of starting lithium, gathering additional information on patient variables and reasons for asking or not asking an investigation would have given some more insight into issues related to monitoring, and poor documentation in medical records.

Few steps that can be taken to improve the monitoring are discussing with colleagues about the issues in following existing protocols, to formulate own standard protocol which is not only feasible to their setup but also evidence based, attaching such a protocol to the medical records of patients on lithium, insisting on strictly adhering to it and proper documentation.

\section{CONCLUSION}

Lithium is one of the commonly used drugs in psychiatry, which has a wide therapeutic use but can be life threatening at toxic levels and can cause serious side effects if not monitored properly. Despite existence of standard guidelines the monitoring in lithium treated patients is 


\section{Standards of Lithium Treatment Monitoring In a Teaching Hospital in South India among Bipolar Affective Disorder}

still at a sub optimal level; hence addressing the issues in monitoring and following a standard protocol can improve the effectiveness of treatment and quality of life of patients.

\section{Acknowledgements}

The author appreciates all those who participated in the study and helped to facilitate the research process.

Conflict of Interests: The author declared no conflict of interests.

\section{REFERENCES}

1. Rihmer Z, Angst J. Mood disorders epidemiology. In: Sadock BJ, Sadock VA, editors. Kaplan \&Sadock's Comprehensive textbook of psychiatry. $8^{\text {th }}$ ed. Philadelphia: LWW; 2005.pp. 1575-82.

2. Jefferson JW, Greist JH. Lithium. In:Sadock BJ, Sadock VA, editors. Kaplan \&Sadock's Comprehensive textbook of psychiatry. $8^{\text {th }}$ ed. Philadelphia: LWW; 2005.pp. 2839-51.

3. Ellenhorn MJ, Lithium. In: Ellenhorn's medical toxicology: diagnosis and treatment of Human poisoning. Baltimore: Williams \& Wilkins; 1997. pp 1579- 85.

4. Goodwin FK, Jamison KR, editors. Manic-Depressive Illness. New york: Oxford University Press; 1990.

5. Johnston AM, Eagles JM. Lithium - associated clinical hypothyroidism: prevalence and risk factors. Br J psychiatry 1999; 175:336-39.

6. Anon. Drug treatments for bipolar disorder: maintenance, prevention and special situations. Drug and Therapeutics Bulletin 2005; 43:33-37.

7. Bendz H, Sjodin I, Aurell M. Renal function on and off lithium in patients treated with lithium for 15 years or more: acontrolled, prospective lithium - withdrawal study. Nephrol Dial Transplant 1996; 11:457-60.

8. National Institute for Clinical Excellence. Principles for Best Practice in Clinical Audit. Radcliffe Medical Press; 2002. Available from: http://www.nice.org.uk/media/796/23/BestPracticeClinicalAudit.pdf.

9. Royal College of Psychiatrists. A Competency Based Curriculum for Specialist Training in Psychiatry: Specialist Module in General Psychiatry. Royal College of Psychiatrists, 2009.

10. Taylor D, Paton C, Kapur S, editors. The Maudsley prescribing guidelines. 10th edition. London: Informa health care; 2009.

11. Collins N, Barnes TRE, Smith AS, Gerrett D, Paton C. Standards of lithium monitoring in mental health trusts in the UK. BMC Psychiatry 2010; 10:80.

12. Glover KJ, Lawley D. How safe is lithium prescribing? Audit of a local prescribing framework and patient survey. Psychiatr Bull 2005; 29:98-100.

13. Kehoe RF, Mander AJ. Lithium treatment: prescribing and monitoring habits in hospital and general practice. BMJ 1992; 304:552-54.

14. Fielding S, Kerr S, Godber C. Lithium in the over-65s-a dedicated monitoring service leads to a better quality of treatment supervision. Int J Geriatr Psychiatry 1999; 14:985-87.

(C) The International Journal of Indian Psychology, ISSN 2348-5396 (e)| ISSN: 2349-3429 (p) | 144 
15. Eagles JM, McCann I, MacLeod TN, Paterson N. Lithium monitoring before and after the distribution of clinical practice guidelines. ActaPsychiatrScand2000; 101:349-53.

16. Holmes B. If you want a job done properly, do it yourself - setting up a county-wide lithium database. Bipolar Disorders 2003; (5, Suppl 1):53.

17. Holmes B. The Norfolk-wide lithium database: the answer, or more questions? Bipolar Disorders 2005; (7,Suppl 2):65.

How to cite this article: Ramasamy S, Srinivasan S, \& Ramanathan S A (2017). Standards of Lithium Treatment Monitoring In a Teaching Hospital in South India among Bipolar Affective Disorder. International Journal of Indian Psychology, Vol. 5, (1), DIP: 18.01.098/20170501, DOI: 10.25215/0501.098 